\title{
Acquisition of Heart rate using NI-myRIO
}

\author{
A. Abirami ${ }^{1}$, P. N. Vasantha ${ }^{2}$, N. Gayathri ${ }^{3}$, G.Vijayalakshmi ${ }^{4}$ \\ U.G Student, Saranathan College of Engineering, Trichy, India ${ }^{1,2,3}$ \\ Assistant Professor, Saranathan College of Engineering, Trichy, India ${ }^{4}$
}

\begin{abstract}
In this new era of machines replacing human, the latter are prone to various kinds of health hazards. This has a direct relevance to the health of a human being and functioning of the heart. Thus, it is of utmost importance to check and maintain the pulse rate under control. This has been achieved by obtaining the heart rate using a PPG (Photoplethysmography) sensor called TCRT 1000 . This paper primarily intends to observe pulse rate using NI myRIO and display the pulse rate graphically using LabVIEW programming.
\end{abstract}

Abiding to the principles of photo-plethysmograph technique, this paper provides a solution to the acquire real time signal from the fingertip of the human. The finger tips are highly receptive and responsive to blood infusions. For every systolic and diastolic pulse changes in the heart, there is a continuous inflow and outflow of blood in the finger tip. The real time signal that has been obtained from the sensor is sent to the virtual environment using the analog input port of NI myRIO. This paper helps in obtaining the output of sensor in digital and graphical format.

Keywords: Pulse rate, LabVIEW, NI myRIO, TCRT1000, Photo-plethysmography (PPG)

\section{INTRODUCTION}

In current scenario, there is utmost risk of heart related syndromes due to change in the food habits and living style. The heart is a muscular organ in both humans and other wildlife, which drives blood through the blood vessels in the circulatory system. Blood supplies the body with oxygen and nutrients, and also assists in the removal of metabolic trashes. Generally with each heartbeat, the right ventricle pumps the same amount of blood into the lungs as the left ventricle pumps out into the body.

Normally at rest, the heart contracts at a rate of around 72 beats per minute. The SA node would create a sinus rhythm of approximately $100 \mathrm{bpm}$ without any nervous stimulation. The relaxing heart rate of a newborn baby can be 120 beats per minute (bpm) and this gradually decreases until adulthood and then gradually rises over with age. The adult resting heart rate ranges from 60-100 bpm. Exercise and fitness levels, age and basal metabolic rate can all affect the heart rate. An athlete's heart rate can be lower than $60 \mathrm{bpm}$. During exercise, the rate can be $150 \mathrm{bpm}$ with maximum rates reaching from 200 and 220 bpm.

\section{SYSTEM OPERATION}

The operation of this paper is well explained using the flow chart of operation and representation in block diagram.

The flow chart described below in figure 1, illustrates the interfacing of real world signal with the virtual environment LabVIEW. Pulse rate (PPG) signal is acquired with the help TCRT1000 pulse rate sensor. The acquired pulse rate signal is sent to NI myRIO with the help of analog pins in the NI myRIO, here pins $7 \& 8$ of the $\mathrm{C}$-port are configured for transferring signal. Then, the myRIO is connected to a PC installed with LabVIEW software. The analog input express VI is connected with an indicator called the waveform chart to output the pulse rate and view it both in numeric digital format and in the form of chart [1]. 


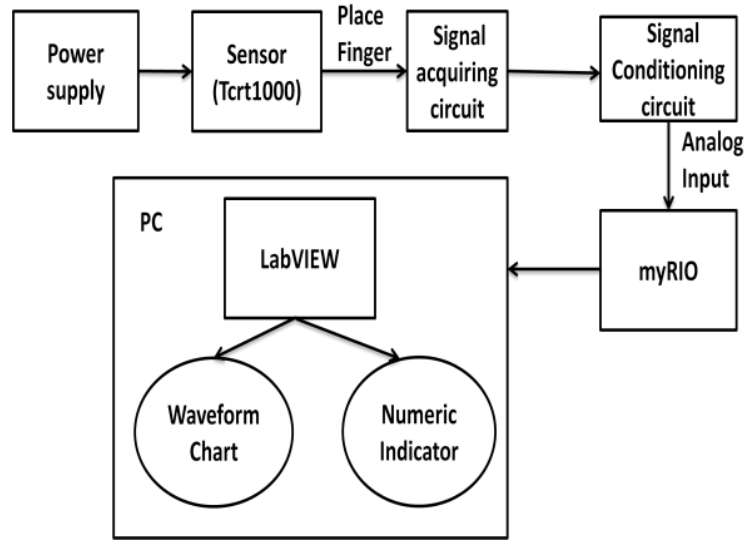

Fig. 2. Block Diagram

\section{DESIGN OF SYSTEM SOFTWARE}

\section{A. LabVIEW}

LabVIEW is abbreviated as Laboratory Virtual Instrumentation Engineering Workbench. It is National Instruments software which will support graphical programming to solve the problems. LabVIEW being a graphical programming language which is extremely supportive to engineering and research field. This virtual platform is largely helpful to resolve complex problems using the data flow execution. This will support the many data types like arrays and clusters and also the loops and structures.

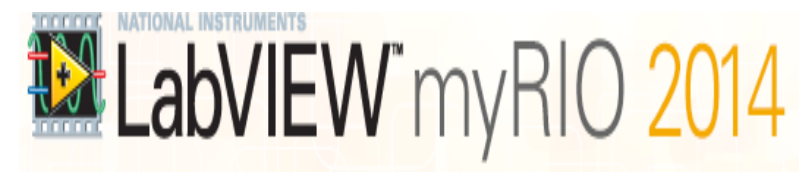

Fig. 3. LabVIEW 2014 Logo

LabVIEW environment has a front panel and block diagram, which is used to create a program in virtual environmental field. In addition to that, tools palette and finding function are also present in this software. This will also support the audio input and audio output.

With the help of data acquisition devices like NI-DAQ, NI-myRIO, Simulated Data Acquisition and Sound Card in laptops the real time signal is deployed into LabVIEW programming. It will give greater application in the fields like signal and image processing, embedded systems programming, simulation and prototyping and also in audio application.

\section{TOOLS USED}

\section{A. HARDWARE COMPONENTS}

\section{- TCRT-1000}

TCRT1000 is a pulse rate monitoring sensor that offers daylight blocking filter, which prevents the external light from affecting the measurement. TCRT1000 is a reflective sensor that comprises of an infrared emitter as transmitter and phototransistor as receiver in a leaded bundle both positioned at a distance of $1 \mathrm{~mm}$.

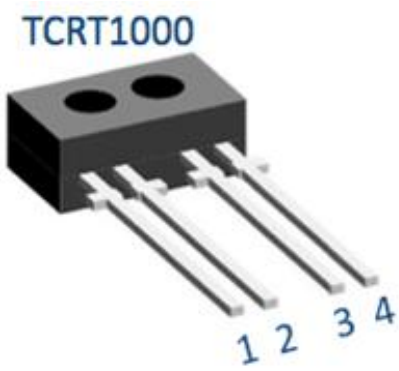

Fig. 4. Sensor TCRT-1000

It is a highly efficient method to obtain signal from the fingertip by the absorbance and reflectance principles. The sensor emits an infrared wavelength of 950nm, which passes through the fingertip and is obtained in the receiver end by the phototransistor.

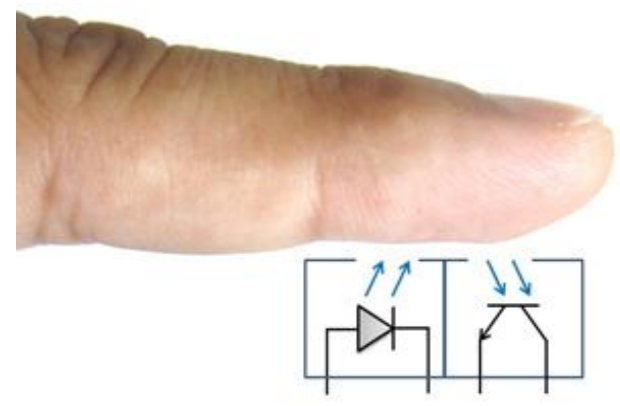

Fig. 5 TCRT-1000 Sensor operation

The basic principle of operation of sensor is that the light emitted from transmitter is influenced by an object or medium on its way to the detector.

The change in the light signal due to this interaction with object produces a proportional change in the electrical signal in the optoelectronic receiver. Thus, TCRT1000 works as a reflex sensor (transmissive sensor).

The PPG signal produced as the output of TCRT1000 consists of two components namely, the AC and the DC component.

The AC component is the area of interest. This signal arises due to the pulsatile changes in the arterial blood volume. Thus, this is the source of heart rate information and is synchronous with the heart beat.

The signal obtained from the sensor is of a small portion of the entire signal. Thus, it requires amplification. Whereas, the DC component refers to the reflectance of light from the tissues in the finger tip,

This directly relates to average blood volume. Generally, the AC component is superimposed with the DC component. Thus, to obtain an AC waveform with high signal-to-noise ratio, it is very important to remove the DC component [2]. 


\section{- $\quad$ NI myRIO}

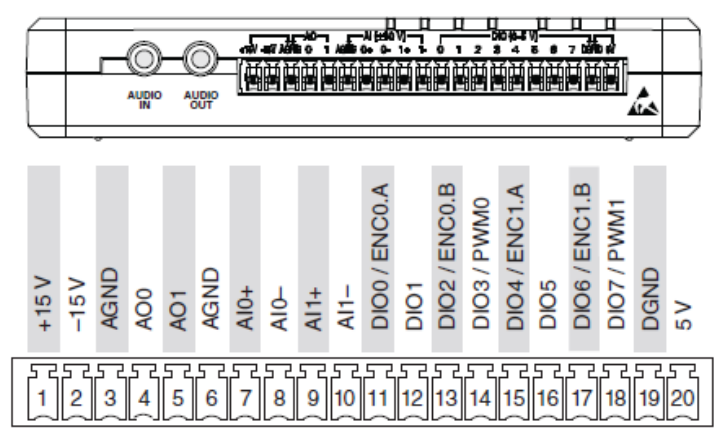

Fig. 6. NI myRIO port $\mathrm{C}$

The National Instruments myRIO is a portable reconfigurable input, output (RIO) device. This will provides analog input (AI), analog output (AO), digital input (DI) and digital output (DO), audio, and power output in a compact embedded device. The NI myRIO connects to a host computer over USB and wireless. This will have two myRIO expansion port and one mini system port. This will support the Learners to use to design control, robotics, and mechatronics systems [3].

Fig. 7. NI myRIO

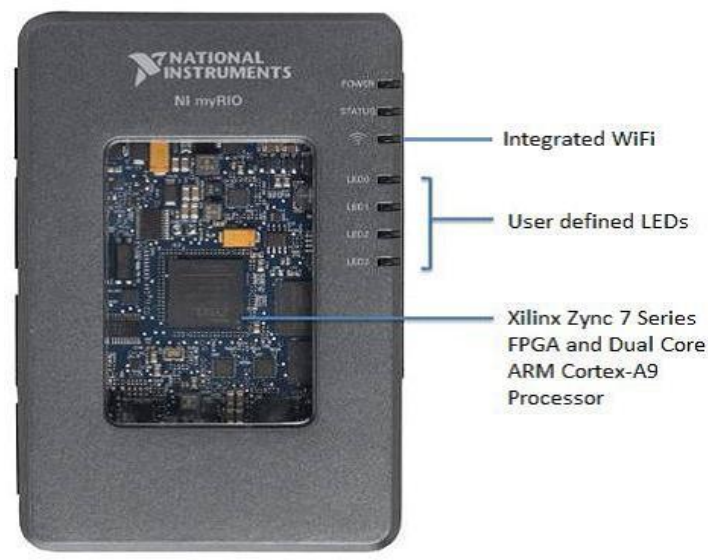

\section{B. SOFTWARE TOOLS}

\section{- TIMED LOOP}

It is a control loop structure statement that allows the program to get repeated after a certain interval of time, the default being $1000 \mathrm{~ms}$.

The timed loop has a lot of advantages over the other control loops.

They are multirate timing capabilities, precise timing, feedback on loop execution, timing characteristics that change dynamically and several levels of execution priority.

The Timed Loop includes one Input and four Output nodes, and two Left and three Right Data nodes for each frame. They have been used to perform deterministic counter scale operations. in the circuit [4].
Fig. 8. Timed loop

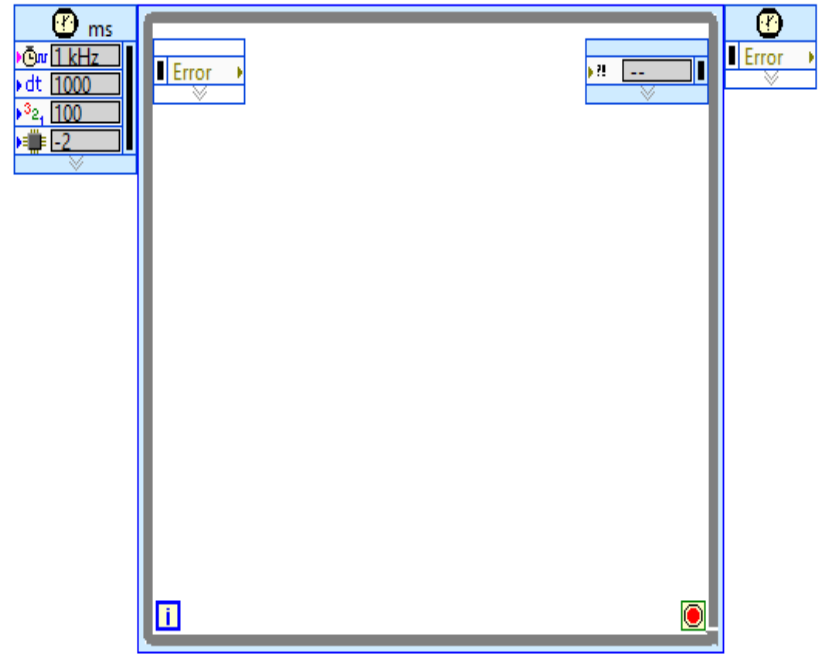

\section{WAVEFORM CHART}

It is a special type of numeric indicator that displays one or more plots of data typically acquired at a constant rate.

It maintains a history of data even from the previous updates A default of about 1024 data points are considered as the chart history length.

It is useful in obtaining the output of the analog real time signal from the sensor to the virtual environment of the LabVIEW coding [3].

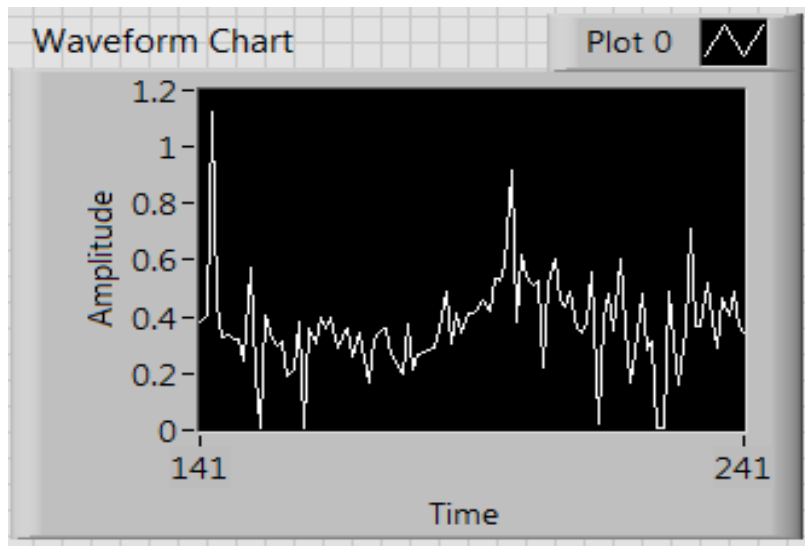

Fig. 9. Waveform chart

\section{RESULT}

The figure 9 represents the output of the discussed paper.

The analog input from the signal conditioning unit is directly connected with myRIO analog pins (7\&8configured) and that signal is acquired with the help of Graphical Programming in LabVIEW.

The real time signal has been obtained in the virtual environment using the Express VI myRIO analog output. The acquired signal is depicted on the waveform chart. 


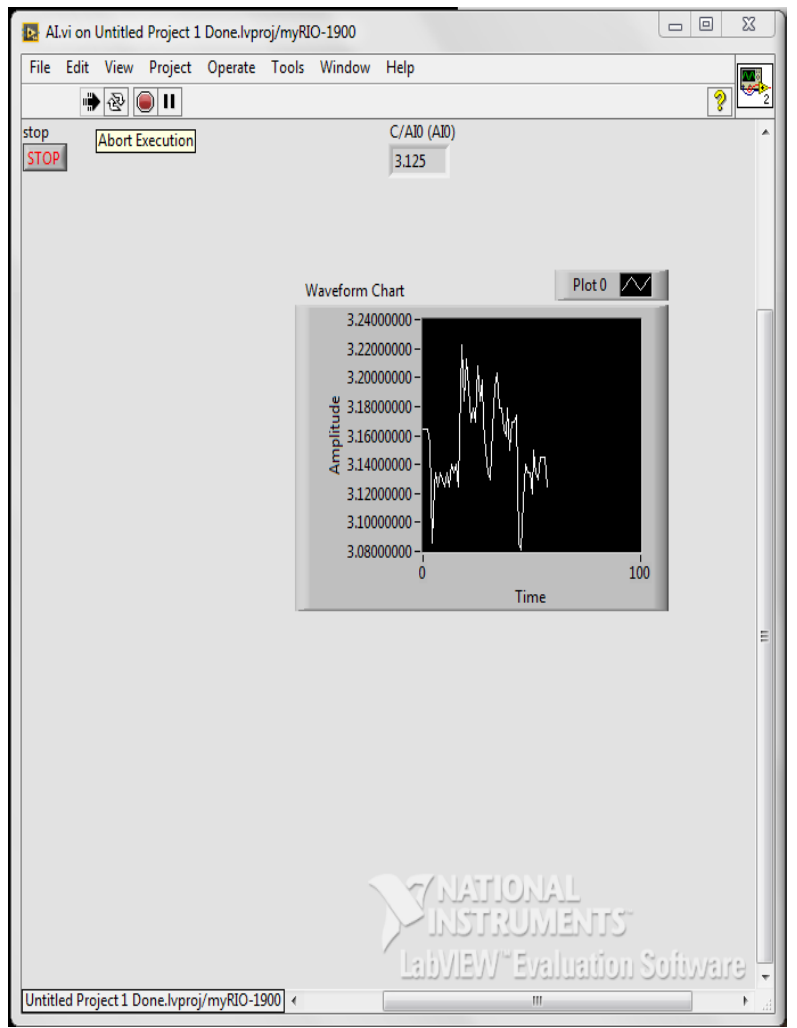

Fig. 10. Output

The power supply given to the acquiring circuit is directly obtained on the chart. Once our fingertip is placed in front of the sensor the supply voltage is reduced. With the help of numeric pallet, the individual output as voltage values are displayed and for graphical representation a waveform chart has been used.

\section{CONCLUSIONS}

From the above investigation, we have interfaced the TCRT1000 sensor with myRIO by acquiring the real time analog signals with the help of photoplethysmographic technique. The output from the sensor is in the form of voltage values that is directly proportional to the absorbance and the reflectance of infrared light from the sensor. The obtained signal is then graphically represented in waveform chart.

\section{ACKNOWLEDGEMENT}

With a deep and profound sense of gratitude, we express our hearty thanks to Ms. G. Vijayalakshmi, Assistant Professor, Instrumentation and Control engineering, Saranathan College of engineering for her excellent guidance and valuable advice throughout this venture.

\section{REFERENCES}

[1] "Design of strap on cardiac peril vigilance system" by, G.Shanmuga Priya, Shruti Srinath and R.Vidhya. Proceedings of 4th IRF International Conference, Chennai, 9th March-2014, ISBN: 978-9382702-64-1

[2] Hashem, M.M.A., Shams, R. Kader, M.A. Sayed, M.A., "Design and development of a heart rate measuring device using fingertip," International Conference on Computer and Communication Engineering, pp. 605-635, 2010.

[3] NI myRIO manual available at www.ni.com/myrio.
[4] NI LabVIEW from www.ni.com

[5] Labview - software www.ni.com/labview/whylabview

[6] Heart rate monitor from Wikipedia. used

available at 